\title{
Spectral properties and conformal type of surfaces
}

\author{
PHILIPPE CASTILLON \\ G.T.A. (U.M.R. 5030), Univ. Montpellier 2, 34095 Montpellier Cedex 5, France \\ Manuscript received on July 26, 2002; accepted for publication on August 30, 2002; \\ presented by MANFREDO DO CARMO
}

\begin{abstract}
In this short note, we announce a result relating the geometry of a riemannian surface to the positivity of some operators on this surface (the operators considered here are of the form surface Laplacian plus a scalar multiple of the curvature function). In particular we obtain a theorem "à la Huber": under a spectral hypothesis we prove that the surface is conformally equivalent to a Riemann surface with a finite number of points removed.

This problem has its origin in the study of stable minimal surfaces.
\end{abstract}

Key words: spectral theory, minimal surfaces, stability operator.

\section{INTRODUCTION}

Minimal submanifolds are solution of a variational problem: they are critical points of the volume functional for deformations with compact support. The second derivative of the volume functional is given by a quadratic form associated to a selfadjoint operator (the stability operator). A minimal immersion is called stable when it is a local minimum of the volume functional, that is when the stability operator is a positive operator.

For a minimal surface $M$ in $\mathbb{R}^{3}$, the stability operator is given by $S=\Delta+2 \mathcal{K}$, where $\mathcal{K}$ is the (intrinsic) curvature of $M$. For a surface immersed in a manifold with nonnegative scalar curvature, the positivity of the stability operator implies the positivity of the operator $L=\Delta+\mathcal{K}$. In order to study stable minimal surfaces, these remarks lead the authors of Fischer-Colbrie and Schoen (1980) to consider the problem of relating the positivity of operators of the form $\Delta+\lambda \mathcal{K}(\lambda \in \mathbb{R})$ on a surface to the geometry of the surface.

Let $(M, h)$ be a complete noncompact Riemannian surface and let $\mathcal{K}$ be its curvature. For all $\lambda \in \mathbb{R}$, consider the operator $L_{\lambda}=\Delta+\lambda \mathcal{K}$ and $q_{\lambda}$ the associated quadratic form. It is easy to see (cf. Fischer-Colbrie and Schoen 1980) that the set $I_{h}=\left\{\lambda \in \mathbb{R} \mid q_{\lambda}\right.$ positive $\}$ is a closed interval:

E-mail: cast@math.univ-montp2.fr 
$I_{h}=\left[a_{h}, b_{h}\right]$ with $-\infty \leq a_{h} \leq 0 \leq b_{h} \leq+\infty$. The general problem is to find relations between the geometry of $M$ and the numbers $a_{h}$ and $b_{h}$.

In Fischer-Colbrie and Schoen (1980), the authors asked the following question: On the disc $D=\{z \in \mathbb{C}|| z \mid<1\}$, consider the complete metrics which are conformal to the Euclidean one; for such a metric $h$, what are the possible values of $b_{h}$ which can occur? As a first step to answer this question, they remark that $b_{h}=1 / 4$ if $h$ is the Poincaré metric and they prove that $b_{h}<1$ for a complete conformal metric on $D$ (cf. Fischer-Colbrie and Schoen 1980: remark 1 and theorem 2).

The purpose of this note is to give the great lines of the proof of the following result which answers the question.

Theorem A. Let $(M, h)$ be a complete noncompact Riemannian surface. If $b_{h}>1 / 4$ then $M$ is conformally equivalent to $\mathbb{C}$ or $\mathbb{C}^{*}=\mathbb{C} \backslash\{0\}$.

A straightforward corollary is that $b_{h} \leq 1 / 4$ when $h$ is a complete conformal metric on the disc. Moreover, the inequality in the statement of theorem $\mathrm{A}$ is optimal since $b_{h}=1 / 4$ for the Poincaré metric on the disc. Notice also that $\mathbb{C}$ and $\mathbb{C}^{*}$ admit complete flat metrics for which $b_{h}=+\infty$.

On the other hand, with a similar proof we get the following theorem which is to be compared with Huber's theorem (cf. Huber 1957):

THEOREM B. Let $(M, h)$ be a complete noncompact Riemannian surface. If there exists a compact domain $\Omega \in M$ and a real $\lambda>1 / 4$ such that $q_{\lambda}(u) \geq 0$ for every $C^{\infty}$-function $u$ with compact support in $M \backslash \Omega$, then $M$ is conformally equivalent to a compact Riemann surface minus a finite number of points.

In this short communication we only give the great lines of the proofs. Detailed proofs will appear elsewhere.

\section{SKETCH OF PROOFS}

\section{NOTATIONS}

Let $x_{0}$ be a point in $M$. In the sequel we shall note $r(x)=d_{M}\left(x_{0}, x\right)$ the distance function to $x_{0}$, $B_{s}=\{x \in M \mid r(x)<s\}$ the ball of radius $s$, and $C_{s}^{t}=\{x \in M \mid s<r(x)<t\}$.

Moreover, we shall note $V(s)$ the volume of the ball $B_{s}, \ell(s)$ the length of the geodesic circle of radius $s$ (i.e. $\left.\ell(s)=\operatorname{vol}\left(\partial B_{s}\right)\right)$ and $G(s)$ the total curvature of the ball $B_{s}$ (i.e. $\left.G(s)=\int_{B_{s}} \mathcal{K} d v_{h}\right)$. Using the coarea formula, we easily have $V^{\prime}(s)=\ell(s)$ and $G^{\prime}(s)=\int_{\partial B_{s}} \mathcal{K} d \sigma_{s}$ where $d \sigma_{s}$ is the volume form on $\partial B_{s}$.

\section{Sketch of Proof of Theorem A}

Using the fact that $q_{\lambda}$ is a positive form for some $\lambda>1 / 4$, the proof consists in estimating $q_{\lambda}(f)$ for suitable functions $f$ (with compact support) of the form $f=\xi(r)$. To do this, we have to 
handle with terms of the form $\int_{C_{R}^{Q}} \mathcal{K} \xi(r)^{2} d v_{h}$. These terms can be estimated using the following technical lemma:

Lemma 1. Let $R<Q$, and let $\xi:[R, Q] \rightarrow \mathbb{R}$ such that $\xi(Q)=0, \xi \geq 0$, $\xi^{\prime} \leq 0$ and $\xi^{\prime \prime} \geq 0$. If there exists a constant $A$ such that $\chi\left(B_{s}\right) \leq A$ for all $s \in[R, Q]$, then

$$
\int_{C_{R}^{Q}} \mathcal{K} \xi(r)^{2} d v_{h} \leq-\xi(R)^{2} G(R)-2 \xi(R) \xi^{\prime}(R) \ell(R)+2 \pi A \xi(R)^{2}-\int_{C_{R}^{Q}}\left(\xi^{2}\right)^{\prime \prime}(r) d v_{h} .
$$

The proof of this lemma is based on the method used by Colding and Minicozzi (2002) which makes a heavy use of the function $\ell(s)$. In Colding and Minicozzi (2002), the authors use this function as if it were absolutely continuous, which is not the case in general. However, this difficulty can be bypassed using the following two properties of $\ell$ (cf. Shiohama and Tanaka (1989) and Shiohama and Tanaka (1993) for proofs of these two facts):

\section{THEOREM 2. The function $\ell$ is differentiable almost everywhere and}

i. for almost all $s \in \mathbb{R}, \ell^{\prime}(s) \leq 2 \pi \chi\left(B_{s}\right)-G(s)$;

ii. for all $a<b, \ell(b)-\ell(a) \leq \int_{a}^{b} \ell^{\prime}(s) d s$.

With the lemma 1 in hand, it remains to use suitable functions $\xi$. The first step consists in controling the topology: under the hypothesis of theorem A we prove that $M$ is of finite topology and that $\chi(M) \geq 0$. The second step consists in controling the volume growth unsing a different function $\xi$ : we prove that there exists a constant $c_{M}$ such that $\operatorname{vol}\left(B_{s}\right) \leq c_{M} s^{2}$. Then it is a standart argument in potential theory that a surface with quadratic volume growth must be parabolic (ie. each end must be conformally equivalent to the punctured disc).

The proof of theorem B follows the same steps, but since the operator $L_{\lambda}$ is assumed to be positive only outside a compact set $\Omega$, we have to use functions of the form $f=\xi(r)$ with compact support outside $\Omega$. The consequence is that we can only prove that the topology is finite, without estimating $\chi(M)$.

\section{SOME REMARKS}

In order to give a complete answer to the question stated in [3], we have to see what happens for $\lambda \in\left[0, \frac{1}{4}\right]$.

Consider a conformal metric $h=\mu^{2}|d z|^{2}$ on the unit disc, let $\alpha \in \mathbb{R}_{+}$, and let $h_{\alpha}=\mu^{2 \alpha}|d z|^{2}$ be a new conformal metric on $D$. Writing the forms $q_{\lambda}$ for these two metrics in terms of $\mu, \alpha$ and the Euclidean metric, it is easy to see that, when the metric $h_{\alpha}$ is complete, we have $b_{h_{\alpha}}=\frac{1}{\alpha} b_{h}$.

If $h$ is the Poincaré metric (ie. $\left.\mu(z)=\frac{2}{1-|z|^{2}}\right)$, then $h_{\alpha}$ is complete for $\alpha \geq 1$ and $b_{h_{\alpha}}=\frac{1}{4 \alpha}$. Moreover, for the metric $h_{\infty}=\mathrm{e}^{\mu(z)}|d z|^{2}$ it is not hard to see that $b_{h_{\infty}}=0$. Thus, we have the following proposition: 
Proposition 3. For all $\lambda \in\left[0, \frac{1}{4}\right]$ there exists a complete conformal metric $h$ on the unit disc such that $b_{h}=\lambda$.

Another natural question is to know wether the value $b_{h}=1 / 4$ is characteristic of the Poincare metric. The answer is negative, and the following proposition give a sufficient condition for a complete conformal metric $h$ on the unit disc to satisfy $b_{h}=1 / 4$ :

PROPOSITION 4. There exists a universal constant $\varepsilon$ such that for all complete conformal metric $h$ on the unit disc satisfying

$$
\mathcal{K} \leq-1 \text { and } \int_{D}|\mathcal{K}+1|^{\frac{3}{2}} d v_{h} \leq \varepsilon
$$

we have $b_{h}=1 / 4$.

The proof of this proposition is based on a theorem by E. Lieb (cf. for example Castillon 2002: theorem 1.3).

\section{RESUMO}

Nesta comunicação, anunciamos um resultado que relaciona a geometria de uma superfície riemanniana com a positividade de certos operadores na superfície (os operadores considerados têm forma "Laplaciano mais um múltiplo da curvatura"). Em particular, obtemos um teorema “à la Huber”': usando uma condição espectral, provamos que a superfície é conformemente equivalente a uma superfície de Riemann menos um número finito de pontos. Este problema tem origem no estudo das superfícies mínimas estáveis.

Palavras-chave: teoria espectral, superfícies mínimas, operador de estabilidade.

\section{REFERENCES}

Castillon P. 2002. Problèmes de petites valeurs propres sur les surfaces de courbure moyenne constante. Proc Amer Math Soc 130: 1153-1163.

Colding T And Minicozzi W. 2002. Estimates for parametric elliptic integrands. Int Math Res Not 6: 291-297.

FisCHER-COLBRIE D AND SCHOEN R. 1980. The structure of complete stable minimal surfaces in 3-manifolds of non-negative scalar curvature. Commun Pure Appl Math 33: 199-211.

Huber A. 1957. On subharmonic functions and differential geometry in the large. Comment Math Helv 32: $13-72$.

Shiohama K and Tanaka M. 1989. An isoperimetric problem for infinitely connected complete open surfaces. In: Geometry of manifolds, Coll Pap 35th Symp Differ Geom, Matsumoto/Japan 1988, Perspect Math 8: 317-343.

Shiohama K and Tanaka M. 1993. The length function of geodesic paralel circle. In: Shiohama K (Ed.) Progress in differential geometry. Adv Stud Pure Math 22: 299-308. 